\title{
Asymptotic Behavior of A Stochastic Quarantine Model
}

\author{
Yadan Li \\ College of Science, University of Shanghai for Science and Technology, Shanghai, P. R. China \\ Email: liyadanmail@126.com
}

\begin{abstract}
In this paper, we consider a stochastic SIQS epidemic model by introducing random fluctuations. Then we present the disease extinction. Moreover, we investigate the stochastically asymptotic behavior of this model with use of the Markov semigroups theory. Finally, we give some numerical simulations to illustrate our mathematical findings.
\end{abstract}

Keywords: Markov semigroups, quarantine, stochastic model, asymptotic behavior.

\section{Introduction}

Quarantine is a commonly used measure to govern the spread of infection qualitatively and quantitatively. For a long time, mathematical models have become important tools for disease control. There are many scholars focusing on epidemic models with quarantine (see $[1,2,3,4]$ ). The classics SIS epidemic model with quarantine was studied by Herbert and Ma [5] as follows

$$
\left\{\begin{array}{l}
S^{\prime}=\Lambda-\beta S I-d S+\gamma I+\varepsilon Q \\
I^{\prime}=\beta S I-(d+\alpha+\delta+\gamma) I \\
Q^{\prime}=\delta I-(d+\alpha+\varepsilon) Q
\end{array}\right.
$$

In this model all parameter values are nonnegative $(\Lambda, \beta, d>0)$ and summarized as follows:

$\Lambda$ : the influx of new members into the population per unit time;

$\beta$ : the transmission coefficient between compartments $\mathrm{S}$ and I;

$\delta$ : the proportional coefficient of quarantined;

$d$ : the natural death rate for S, I, Q compartments;

$\gamma$ : the recovery rate;

$\varepsilon$ : the rate of losing their immunity for quarantined individuals;

$\alpha$ : the death rate caused by disease.

And $S, I, Q$ denote the numbers of susceptible individuals, infected individuals and quarantined individuals, respectively. The basic reproductive number $R_{0}$ of model (1) is

$$
R_{0}=\frac{\Lambda \beta}{d(\gamma+\delta+d+\alpha)}
$$

Let the state space be $\mathbb{X}=\mathbb{R}_{+}^{3}=\{(S, I, Q): S \geq 0, I \geq 0, Q \geq 0\}$, the bounded set $\Gamma=\{(S, I, Q) \in \mathbb{X}$ : $\left.S+I+Q \leq \frac{\Lambda}{d}\right\} \subset \mathbb{X}$. From [5] we have the following results for system (1):

(a) When $R_{0} \leq 1, \Gamma$ is an asymptotic stability region of the disease-free equilibrium $E_{0}=\left(\frac{\Lambda}{d}, 0,0\right)$;

(b) When $R_{0}>1$, the region $\Gamma \backslash\{(S, I, Q) \mid I=0\}$ is an asymptotic stability region of the endemic equilibrium $E^{*}=\left(S^{*}, I^{*}, Q^{*}\right)$, and $E_{0}$ is unstable.

As we all know, there are full of randomness and stochasticity in our real life. Many studies $[6,7,8]$ show that environmental changes have a tremendous impact on the development of epidemic. The stochastic differential equations (SDE) may be a more appropriate way for describing epidemics in many cases $[9,10,11,12]$. For the model (1) the key parameter to disease transmission is the disease transmission coefficient $\beta$. Then we assume that $\beta$ is subject to the environmental white noise, that is

$$
\beta \rightarrow \beta+\sigma \dot{B}_{t},
$$


where $B_{t}$ is a standard Brownian motion and $\sigma^{2}>0$ is the intensity of environmental forcing. Then model (1) becomes

$$
\left\{\begin{array}{l}
d S_{t}=\left[\Lambda-\beta S_{t} I_{t}-d S_{t}+\gamma I_{t}+\varepsilon Q_{t}\right] d t-\sigma S I d B_{t} \\
d I_{t}=\left[\beta S_{t} I_{t}-(d+\alpha+\delta+\gamma) I_{t}\right] d t+\sigma S_{t} I_{t} d B_{t} \\
d Q_{t}=\left[\delta I_{t}-(d+\alpha+\varepsilon) Q_{t}\right] d t
\end{array}\right.
$$

The existence of a stationary distribution of model(2) and its asymptotic stability is studied in this paper.

Denote

$$
R_{0}^{s}=R_{0}-\frac{\Lambda^{2} \sigma^{2}}{2 d^{2}(\gamma+\delta+d+\alpha)}
$$

In addition, the classical stochastic differential equation SIQS model introduced in this paper adopts the method by Mao [13]. The method has been described in $[14,15,16]$, and assumes that the parameters contained in the model fluctuate near a mean value because of the environment's continuous fluctuation. By using the Markov semigroups theory, we demonstrate that the reproduction number $R_{0}^{s}$ can be used to control the stochastic dynamic of SDE model. If $R_{0}^{s}<1$, the SDE system has a disease free absorbing set which means the extinction of disease with probability one. If $R_{0}^{s}>1$, the amplitude of the noise is small enough, it has an endemic stationary distribution which sparks off the stochastical persistence of the disease. In other words, random fluctuations can influence disease outbreak. This provides us some useful strategies to regulate disease dynamics.

The rest of this paper is organized as follows: In Section 2, we present some preliminary knowledges; In Section 3, we present the main results; In Section 4, we make numerical simulation to support our results.

\section{Preliminaries}

To prove our main results we need some auxiliary definitions and results concerning Markov semigroups and asymptotic properties which are present in $[17,18,19,20,21,22]$.

Let $\Sigma$ be the $\sigma$-algebra of Borel subsets of $\mathbb{X}$ and there exists a Lebesgue measure $m$ on $(\mathbb{X}, \Sigma)$. There exists $\mathbb{D}=\mathbb{D}(\mathbb{X}, \Sigma, m)$ the subset for the space $L^{1}=L^{1}(\mathbb{X}, \Sigma, m), \mathbb{D}=\left\{g \in L^{1}: g \geq 0,\|g\|=1\right\}$, where $\|\cdot\|$ stands for the norm in $L^{1}$. If $P(\mathbb{D}) \subset \mathbb{D}$ then the linear mapping $P: L^{1} \rightarrow L^{1}$ is called a Markov operator.

A family $\{P(t)\}_{t \geq 0}$ of Markov operators satisfies the conditions as follows:

(a) $P(0)=I d$;

(b) for $s, t \geq 0, P(t+s)=P(t) P(s)$;

(c) the function $t \mapsto P(t) g$ is continuous for each $g \in L^{1}$.

If for each $t>0 P(t) g *=g *$ then the density $g *$ is invariant. If there exists an invariant density $g *$ such that $\lim _{t \rightarrow \infty}\|P(t) g-g *\|=0$ for $g \in \mathbb{D}$, the Markov semigroup $\{P(t)\}_{t \geq 0}$ is asymptotically stable.

If for every $g \in \mathbb{D}$, it yields $\lim _{t \rightarrow \infty} \int_{A} P(t) g(x) m(d x)=0$, the Markov semigroup $\{P(t)\}_{t \geq 0}$ is sweeping in connection with a set $A \in \Sigma$.

Lemma 2.1. For $t>0$, with a continuous kernel $k(t, x, y)$ set $\{P(t)\}_{t \geq 0}$ is an integral Markov semigroup, which for all $y \in \mathbb{X}$ satisfies $\int_{\mathbb{X}} k(x, y) m(d x)=1$. We assume that for every $g \in \mathbb{D}$ then

$$
\int_{0}^{\infty} P(t) g d t>0
$$

with respect to compact sets this semigroup is asymptotically stable or sweeping.

For the diffusion process $\left(S_{t}, I_{t}, Q_{t}\right)$, by $\mathcal{P}(t, x, y, z, A)$ we denote the transition probability function, i.e. $\mathcal{P}(t, x, y, z, A)=\operatorname{Prob}\left\{\left(S_{t}, I_{t}, Q_{t}\right) \in A\right\}\left(S_{t}, I_{t}, Q_{t}\right)$ is the solution of (2) where a initial condition is 
$\left(S_{0}, I_{0}, Q_{0}\right)=(x, y, z)$. If $t>0$, the distribution of $\left(S_{t}, I_{t}, Q_{t}\right)$ is absolutely continuous and has density $v(x, y, z)$. Then $u(t, x, y, z)$ satisfies the following Fokker-Planck equation $[22,9]$ :

$$
\frac{\partial u}{\partial t}=\frac{1}{2} \sigma^{2}\left(\frac{\partial^{2}(\varphi u)}{\partial x^{2}}+\frac{\partial^{2}(\varphi u)}{\partial x \partial y}+\frac{\partial^{2}(\varphi u)}{\partial y^{2}}\right)-\frac{\partial\left(f_{1} u\right)}{\partial x}-\frac{\partial\left(f_{2} u\right)}{\partial y}-\frac{\partial\left(f_{3} u\right)}{\partial z}
$$

where $\varphi(x, y, z)=x^{2} y^{2}$ and

$$
\begin{aligned}
& f_{1}(x, y, z)=\Lambda-\beta x y-d x+\gamma y+\varepsilon z \\
& f_{2}(x, y, z)=\beta x y-(d+\alpha+\delta+\gamma) y, \\
& f_{3}(x, y, z)=\delta y-(d+\alpha+\varepsilon) z .
\end{aligned}
$$

Connected with (4) now we present a Markov semigroup. For $V(x, y, z) \in \mathbb{D}$, set $P(t) V(x, y, z)=$ $u(x, y, z, t)$. Since $P(t)$ is a contraction on $\mathbb{D}$ of $L^{1}(\mathbb{X}, \Sigma, m)$ it can be extended to a contraction. Consequently, the Markov semigroup is formed by the operators $\{P(t)\}_{t \geq 0}$. Set $\mathcal{A}$ be an infinitesimal generator for the semigroup $\{P(t)\}_{t \geq 0}$, i.e.

$$
\mathcal{A} V=\frac{1}{2} \sigma^{2}\left(\frac{\partial^{2}(\varphi V)}{\partial x^{2}}+\frac{\partial^{2}(\varphi V)}{\partial x \partial y}+\frac{\partial^{2}(\varphi V)}{\partial y^{2}}\right)-\frac{\partial\left(f_{1} V\right)}{\partial x}-\frac{\partial\left(f_{2} V\right)}{\partial y}-\frac{\partial\left(f_{3} V\right)}{\partial z} .
$$

The adjoint operator $\mathcal{A}^{*}$ is

$$
\mathcal{A}^{*} V=\frac{1}{2} \sigma^{2}\left(\frac{\partial^{2}(\varphi V)}{\partial x^{2}}+\frac{\partial^{2}(\varphi V)}{\partial x \partial y}+\frac{\partial^{2}(\varphi V)}{\partial y^{2}}\right)+f_{1} \frac{\partial V}{\partial x}+f_{2} \frac{\partial V}{\partial y}+f_{3} \frac{\partial V}{\partial z}
$$

\section{Main Results}

In this section, we present the main results.

\subsection{Disease Extinction}

Useing the same way as in [23], we can get that for any initial value $\left(S_{0}, I_{0}, Q_{0}\right) \in \Gamma$, there exist a unique global solution for model (2) and the solution will stay in $\Gamma$ whenever it starts. That is:

Theorem 3.1. For any initial value $\left(S_{0}, I_{0}, Q_{0}\right) \in \Gamma$, there exists a unique global solution $\left(S_{t}, I_{t}, Q_{t}\right) \in \Gamma$ for the model (2) on $t \geq 0$ with probability 1 , that is,

$$
\operatorname{Prob}\left\{\left(S_{t}, I_{t}, Q_{t}\right) \in \Gamma, \forall t \geq 0\right\}=1
$$

Theorem 3.2. Let $\left(S_{t}, I_{t}, Q_{t}\right)$ is the solution of model (2) with any given initial value $\left(S_{0}, I_{0}, Q_{0}\right) \in \Gamma$. One of the following conditions satisfied (1) $\frac{\Lambda}{d}<\frac{\beta}{\sigma^{2}}, R_{0}^{s}<1$; (2) $\frac{\Lambda}{d}>\frac{\beta}{\sigma^{2}}, \sigma^{2}>\frac{\beta^{2}}{2(\gamma+\delta+d+\alpha)}$, we have

$$
\begin{aligned}
\limsup _{t \rightarrow \infty} \frac{\ln I_{t}}{t} & \leq-c<0, \text { a.s., } \\
\limsup _{t \rightarrow \infty} \frac{\ln Q_{t}}{t} & \leq \min \{-(d+\alpha+\varepsilon),-c\}, \text { a.s., } \\
\lim _{t \rightarrow \infty} S_{t} & =\frac{\Lambda}{d}, \text { a.s., }
\end{aligned}
$$

where $c=\left\{\begin{array}{l}(d+\alpha+\delta+\gamma)\left(1-R_{0}^{S}\right), \text { when } \frac{\Lambda}{d}<\frac{\beta}{\sigma^{2}}, \\ (d+\alpha+\delta+\gamma)-\frac{\beta^{2}}{2 \sigma^{2}}, \text { when } \frac{\Lambda}{d}>\frac{\beta}{\sigma^{2}} .\end{array}\right.$ In other words, the disease dies out in probability one, 
Proof. By Itô's formula, we have

$$
\begin{aligned}
d \ln I & =\frac{1}{I}-\frac{1}{2 I^{2}}(d I)^{2} \\
& =\phi(S, I) d t+\sigma S d B_{t}
\end{aligned}
$$

where $\phi: \mathbb{R}_{+}^{2} \rightarrow \mathbb{R}$ is defined by

$$
\phi(S, I)=\beta S-(d+\alpha+\delta+\gamma)-\frac{1}{2} \sigma^{2} S^{2} .
$$

Hence,

$$
\ln I_{t}=\ln I_{0}+\int_{0}^{t} \phi\left(S_{s}, I_{s}\right) d s+\int_{0}^{t} \sigma S_{s} d B_{s}
$$

set $G(t)=\int_{0}^{t} \sigma S_{s} d B_{s}$ implies that

$$
\frac{\langle G, G\rangle_{t}}{t}=\frac{1}{t} \int_{0}^{t} \sigma^{2} S_{s}^{2} d s \leq \sigma^{2} \frac{\Lambda^{2}}{d^{2}}<+\infty .
$$

By the large numbers of martingale laws [9], we get $\limsup _{t \rightarrow \infty} \frac{G(t)}{t}=0$ a.s..

We can get

if $\frac{\Lambda}{d}<\frac{\beta}{\sigma^{2}}$,

$$
\begin{aligned}
\phi(S, I) & =\beta S-(d+\alpha+\delta+\gamma)-\frac{1}{2} \sigma^{2} S^{2} \\
& =-\frac{1}{2}\left(\sigma S-\frac{\beta}{\sigma}\right)^{2}+\frac{\beta^{2}}{2 \sigma^{2}}-(d+\alpha+\delta+\gamma) \\
& \leq-\frac{1}{2} \sigma^{2}\left(\frac{\Lambda}{d}\right)^{2}+\beta \frac{\Lambda}{d}-(d+\alpha+\delta+\gamma) \\
& =(d+\alpha+\delta+\gamma)\left(R_{0}^{S}-1\right),
\end{aligned}
$$

if $\frac{\Lambda}{d}>\frac{\beta}{\sigma^{2}}$,

$$
\begin{aligned}
\phi(S, I) & =\beta S-(d+\alpha+\delta+\gamma)-\frac{1}{2} \sigma^{2} S^{2} \\
& =-\frac{1}{2}\left(\sigma S-\frac{\beta}{\sigma}\right)^{2}+\frac{\beta^{2}}{2 \sigma^{2}}-(d+\alpha+\delta+\gamma) \\
& \leq-\frac{1}{2} \sigma^{2}\left(\frac{\beta}{\sigma^{2}}\right)^{2}+\beta \frac{\beta}{\sigma^{2}}-(d+\alpha+\delta+\gamma) \\
& =\frac{\beta^{2}}{2 \sigma^{2}}-(d+\alpha+\delta+\gamma) .
\end{aligned}
$$

It then follows from (7) that

$$
\ln I_{t} \leq \ln I_{0}-c t+G(t) .
$$

Dividing by $t$ on the both sides of above inequality and letting $t \rightarrow \infty$ we can get that there exist some null set $\mathcal{N}_{1}$ so that $\operatorname{Prob}\left(\mathcal{N}_{1}\right)=1$ and for any $\omega \in \mathcal{N}_{1}$,

$$
\limsup _{t \rightarrow \infty} \frac{\ln I_{t}}{t} \leq-c<0
$$

Thus for any sufficiently small $\eta_{1}>0$, there exists $T_{1}=T_{1}(\omega)$ so that

$$
I_{t}(\omega) \leq e^{\left(-c+\eta_{1}\right) t}, \forall t \geq T_{1}
$$


Therefore, following from the third equation of the model (2), for all $\omega \in \mathcal{N}_{1}$, if $t \geq T_{1}(\omega)$,

$$
\begin{aligned}
Q_{t}(\omega) & =e^{-(d+\alpha+\varepsilon) t}\left(\delta \int_{0}^{t} e^{(d+\alpha+\varepsilon) s} I_{s} d s+Q_{0}\right) \\
& \leq Q_{0} e^{-(d+\alpha+\varepsilon) t}+\delta e^{-(d+\alpha+\varepsilon) t} \int_{0}^{T_{1}} e^{(d+\alpha+\varepsilon) s} I_{s} d s+\delta e^{-(d+\alpha+\varepsilon) t} \int_{T_{1}}^{t} e^{\left(d+\alpha+\varepsilon-c+\eta_{1}\right) s} d s .
\end{aligned}
$$

It follows that for any $\omega \in \mathcal{N}_{1}$

$$
\limsup _{t \rightarrow \infty} \frac{1}{t} \ln Q_{t}(\omega) \leq \min \left\{-(d+\alpha+\varepsilon),-c+\eta_{1}\right\}
$$

Let $\eta_{1} \rightarrow 0$, we get

$$
\limsup _{t \rightarrow \infty} \frac{1}{t} \ln Q_{t}(\omega) \leq \min \{-(d+\alpha+\varepsilon),-c\} \equiv-\lambda
$$

Thus for any sufficiently small $\eta_{2}>0$ and $\omega \in \mathcal{N}_{1}$, there exists $T_{2}=T_{2}(\omega)$ such that

$$
Q_{t}(\omega) \leq e^{\left(-\lambda+\eta_{2}\right) t}, \forall t \geq T_{2} .
$$

From model (2), we have $\dot{N}=\Lambda-d N-\alpha(I+Q)$.It follows from (8) and (9) and the Limit Theorem[24] we obtain: the limit equation is $\dot{N}=\Lambda-d N$ and $\lim _{t \rightarrow \infty} N=\frac{\Lambda}{d}$.

Together with (8) and (9), it yields $\lim _{t \rightarrow \infty} S_{t}=\frac{\Lambda}{d}$, a.s. The proof is completed.

\subsection{Stationary Distribution Exists}

Theorem 3.3. Let $\left(S_{t}, I_{t}, Q_{t}\right)$ be the solution of model $(2)$ with any initial value $\left(S_{0}, I_{0}, Q_{0}\right) \in \Gamma$. If $R_{0}^{s}>1$ and $\sigma^{2}<\min \left\{A_{1}, A_{2}, A_{3}\right\}$, where

$$
\begin{aligned}
A_{1} & =\frac{h_{1}}{b_{1}}, A_{2}=\frac{h_{1} h_{2}-b_{2}}{b_{1} h_{2}}, A_{3}=\frac{h_{1} b_{3}-b_{2}}{b_{2} b_{3}}, \\
h_{1} & =2 \varepsilon \beta+2 d \beta(2 d+\alpha), \\
h_{2} & =d+\alpha+\frac{2 d+\alpha}{\varepsilon}(d+\alpha+\delta), \\
b_{1} & =I^{*}(2 d+\alpha)(2 d+\alpha+\varepsilon+\delta), \\
b_{2} & =S^{*^{2}} d \frac{\varepsilon+\alpha+2 d}{\varepsilon}\left(2 \varepsilon \beta(d+1)+\sigma^{2} b_{1}\right), \\
b_{3} & =d+\alpha+\frac{\alpha}{\delta}(d+\alpha+\varepsilon) .
\end{aligned}
$$

Then there exists a unique density $u_{*}(x, y, z)$ which is the steady-state solution for model $(2)$ and

$$
\lim _{t \rightarrow \infty} \iiint_{\mathbb{X}}\left|u(t, x, y, z)-u_{*}(x, y, z)\right| d x d y d z=0 .
$$

Furthermore, we get

$$
\Pi=\operatorname{supp} u_{*}=\left\{(x, y, z) \in \mathbb{X}: \frac{\Lambda}{d+\alpha}<x+y+z<\frac{\Lambda}{d}\right\} .
$$

The strategies for this proof are as follows:

- First, with the use of Hörmander Theorem [25], it shows that the transition function for process $\left(S_{t}, I_{t}, Q_{t}\right)$ is absolutely continuous.

- Then, with the use of support theorems [26,27], on $\mathbb{X}$, it shows that the density for transition function is positive.

- Next, it shows that the ąřFoguel alternativeąś [20] is satisfied for Markov semigroup.

- Finally, by proving there is a Khasminskii function [28] we exclude sweeping.

We realize this strategy by following Lemmas 3.4, 3.5, 3.6, 3.8, 3.9. 
Lemma 3.4. For each point $\left(x_{0}, y_{0}, z_{0}\right) \in \mathbb{X}$ and $t>0, \mathcal{P}(t, x, y, z, A)$ is the transition probability function which has a continuous density $k\left(t, x, y, z ; x_{0}, y_{0}, z_{0}\right)$.

Proof. If $b(x)$ and $c(x)$ are vector fields on $\mathbb{R}^{d}$, then the Lie bracket $[b, c]$ is a vector field given by

$$
[b, c]_{j}(x)=\sum_{k=1}^{d}\left(b_{k} \frac{\partial c_{j}}{\partial x_{k}}(x)-c_{k} \frac{\partial b_{j}}{\partial x_{k}}(x)\right), j=1,2, \cdots, d .
$$

Let

$$
a_{0}(S, I, Q)=\left(\begin{array}{c}
\Lambda-\beta S I-d S+\gamma I+\varepsilon Q \\
\beta S I-(d+\alpha+\delta+\gamma) I \\
\delta I-(d+\alpha+\varepsilon) Q
\end{array}\right), \quad a_{1}(S, I, Q)=\left(\begin{array}{c}
-\sigma S I \\
\sigma S I \\
0
\end{array}\right)
$$

Then, by direct calculating, the Lie bracket $\left[a_{0}, a_{1}\right]$ is a vector field given by

$$
a_{2}=\left[a_{0}, a_{1}\right]=\left(\begin{array}{c}
-\Lambda \sigma I-\sigma \gamma I^{2}-\varepsilon \sigma I Q+(d+\alpha+\delta) \sigma S I \\
\Lambda \sigma I-d \sigma S I+\sigma \gamma I^{2}+\varepsilon \sigma I Q \\
-\sigma \delta S I
\end{array}\right)
$$

and

$$
a_{3}=\left[a_{1}, a_{2}\right]=\left(\begin{array}{c}
-\Lambda \sigma^{2} I^{2}-\sigma^{2} \gamma I^{3}-\varepsilon \sigma^{2} I^{2} Q-\sigma^{2} \gamma S I^{2}+(\alpha+\delta) \sigma^{2} S I^{2} \\
\Lambda \sigma^{2} I^{2}+\sigma^{2} \gamma I^{3}+\varepsilon \sigma^{2} I^{2} Q+\sigma^{2} \gamma S I^{2}-(\alpha+\delta) \sigma^{2} S I^{2} \\
\sigma^{2} \delta S I^{2}-\sigma^{2} \delta S^{2} I
\end{array}\right)
$$

Then $a_{1}, a_{2}, a_{3}$ are linear independent on $\mathbb{X}$. Hence for every $(S, I, Q) \in \mathbb{X}$, vector $a_{1}(S, I, Q), a_{2}(S, I, Q)$, $a_{3}(S, I, Q)$ span the space $\mathbb{X}$. By the Hörmander Theorem, the transfer probability function $\mathcal{P}(t, x, y, z, A)$ has a continuous density $k\left(t, x, y, z ; x_{0}, y_{0}, z_{0}\right), k \in C^{\infty}((0, \infty) \times \mathbb{X} \times \mathbb{X})$.

Then, we present a method which based on support theorems that allows us to check at which the kernel $k$ is positive. Fix a function $\phi \in L^{2}([0, T] ; \mathbb{R})$ and a point $\left(x_{0}, y_{0}, z_{0}\right) \in \mathbb{X}$, considering the following integral equation system:

$$
\left\{\begin{array}{l}
x_{\phi}(t)=x_{0}+\int_{0}^{t}\left(f_{1}\left(x_{\phi}(s), y_{\phi}(s), z_{\phi}(s)\right)-\phi \sigma x_{\phi}(s) y_{\phi}(s)\right) d s \\
y_{\phi}(t)=y_{0}+\int_{0}^{t}\left(f_{2}\left(x_{\phi}(s), y_{\phi}(s), z_{\phi}(s)\right)-\phi \sigma x_{\phi}(s) y_{\phi}(s)\right) d s \\
z_{\phi}(t)=z_{0}+\int_{0}^{t} f_{3}\left(x_{\phi}(s), y_{\phi}(s), z_{\phi}(s)\right) d s .
\end{array}\right.
$$

where $f_{1}(x, y, z), f_{2}(x, y, z), f_{3}(x, y, z)$ are defined as $(5)$.

For function $h \mapsto X_{\phi+h}(T)$ from $L^{2}([0, T] ; \mathbb{R})$ to $\mathbb{X}$, let $D_{X_{0} ; \phi}$ is the Fréchet derivative. We denote $X=(x, y, z)^{T}, X_{0}=\left(x_{0}, y_{0}, z_{0}\right)^{T}$, as for some function $\phi \in L^{2}([0, T] ; \mathbb{R})$ the derivative $D_{X_{0} ; \phi}$ has rank 3 , then for $X=X_{\phi}(T)$ have $k\left(T, x, y, z ; x_{0}, y_{0}, z_{0}\right)>0$. Let

$$
\Psi(t)=f^{\prime}\left(X_{\phi}(t)\right)+\phi g^{\prime}\left(X_{\phi}(t)\right)
$$

where $g^{\prime}$ and $f^{\prime}$ are the Jacobians of $g=\left(\begin{array}{c}-\sigma x y \\ \sigma x y \\ 0\end{array}\right)$ and $f=\left(\begin{array}{l}f_{1}(x, y, z) \\ f_{2}(x, y, z) \\ f_{3}(x, y, z)\end{array}\right)$. And let $H\left(t, t_{0}\right)$, for $0 \leq t_{0} \leq t \leq T$, be a matrix function such that $H\left(t_{0}, t_{0}\right)=I d$ and $\frac{\partial H\left(t, t_{0}\right)}{\partial t}=\Psi(t) H\left(t, t_{0}\right)$. Then $D_{X_{0} ; \phi} h=\int_{0}^{T} H(T, s) g(s) h(s) d s$.

Lemma 3.5. For each $\left(x_{0}, y_{0}, z_{0}\right) \in \Pi$ and $(x, y, z) \in \Pi$, there exists $T>0$ such that $k\left(T, x, y, z ; x_{0}, y_{0}, z_{0}\right)>$ 0 , where $\Pi$ is the same as in (10). 
Proof. Since a continuous control function $\phi$ is considered, model (11) can be removed by the following differential equations model:

$$
\left\{\begin{array}{l}
x_{\phi}^{\prime}(t)=f_{1}\left(x_{\phi}(t), y_{\phi}(t), z_{\phi}(t)\right)-\phi \sigma x_{\phi}(t) y_{\phi}(t), \\
y_{\phi}^{\prime}(t)=f_{2}\left(x_{\phi}(t), y_{\phi}(t), z_{\phi}(t)\right)-\phi \sigma x_{\phi}(t) y_{\phi}(t), \\
z_{\phi}^{\prime}(t)=f_{3}\left(x_{\phi}(t), y_{\phi}(t), z_{\phi}(t)\right) .
\end{array}\right.
$$

First, we prove that the rank of $D_{X_{0} ; \phi}$ is 3 . Let $\eta \in(0, T)$ and $h(t)=\frac{\chi_{[T-\eta, T]}}{x_{\phi}(t) y_{\phi}(t)}, t \in[0, T]$, where $\chi$ is the characteristic function. We obtain

$$
D_{X_{0} ; \phi} h=\eta \mathbf{v}-\frac{\mathbf{1}}{\mathbf{2}} \eta^{2} \mathbf{\Psi}(\mathbf{T}) \mathbf{v}+\frac{\mathbf{1}}{\mathbf{6}} \eta^{\mathbf{3}} \mathbf{\Psi}^{\mathbf{2}}(\mathbf{T}) \mathbf{v}+\mathbf{o}\left(\eta^{3}\right),
$$

where $\mathbf{v}=\left(\begin{array}{c}-\sigma \\ \sigma \\ 0\end{array}\right)$. Compute

$$
\Psi(T) \mathbf{v}=\sigma\left(\begin{array}{c}
(\beta+\phi \sigma)(y-x)+d+\gamma \\
(\beta+\phi \sigma)(x-y)-(d+\alpha+\delta+\gamma) \\
\delta
\end{array}\right), \quad \Psi^{\mathbf{2}}(\mathbf{T}) \mathbf{v}=\sigma\left(\begin{array}{l}
b_{1} \\
b_{2} \\
b_{3}
\end{array}\right),
$$

where

$$
\begin{aligned}
b_{1}= & -(\beta y+d+\phi \sigma y)^{2}-(-\beta x+\gamma-\phi \sigma x)(\beta y+\phi \sigma y)-(\beta y+d+\phi \sigma x)(-\beta x+\gamma-\phi \sigma x) \\
& +(-\beta x+\gamma-\phi \sigma x)(\beta x-d-\alpha-\delta-\gamma+\phi \sigma x)+\varepsilon \delta, \\
b_{2}= & (\beta y+\phi \sigma y)(\beta y+d+\phi \sigma y)-(\beta x-d-\alpha-\delta-\gamma+\phi \sigma x)(\beta y+\phi \sigma y \\
& +(-\beta x+\gamma-\phi \sigma x)(\beta y+\phi \sigma y)+(\beta x-d-\alpha-\delta-\gamma+\phi \sigma x)^{2}, \\
b_{3}= & -\delta(\beta y+\phi \sigma y)+\delta(\beta x-d-\alpha-\delta-\gamma+\phi \sigma x)-\delta(d+\alpha+\varepsilon) .
\end{aligned}
$$

Therefore, it follows that $\mathbf{v}, \Psi(T) \mathbf{v}$ and $\Psi^{2}(T) \mathbf{v}$ are linear independent and the rank for derivative $D_{X_{0} ; \phi}$ is 3 .

Next, we claim that for any two points $X \in \Pi$ and $X_{0} \in \Pi$, there exist $T>0$ and a control function $\phi$ so that $X_{\phi}(0)=X_{0}, X_{\phi}(T)=X$. Let $w_{\phi}=x_{\phi}+y_{\phi}+z_{\phi}$, model (12) becomes

$$
\left\{\begin{aligned}
x_{\phi}^{\prime}(t) & =g_{1}\left(x_{\phi}(t), w_{\phi}(t), z_{\phi}(t)\right)-\phi \sigma x_{\phi}(t)\left(w_{\phi}(t)-x_{\phi}(t)-z_{\phi}(t)\right), \\
w_{\phi}^{\prime}(t) & =g_{2}\left(x_{\phi}(t), w_{\phi}(t), z_{\phi}(t)\right), \\
z_{\phi}^{\prime}(t) & =g_{3}\left(x_{\phi}(t), w_{\phi}(t), z_{\phi}(t)\right) .
\end{aligned}\right.
$$

where

$$
\begin{aligned}
& g_{1}(x, w, z)=\Lambda-\beta x(w-x-z)-d x+\gamma(w-x-z)+\varepsilon z, \\
& g_{2}(x, w, z)=\beta x(w-x-z)-(d+\alpha+\delta+\gamma)(w-x-z), \\
& g_{3}(x, w, z)=\delta(w-x-z)-(d+\alpha+\varepsilon) z .
\end{aligned}
$$

Let

$$
\Pi_{0}=\left\{(x, w, z) \in \mathbb{X}: 0<x, z<\frac{\Lambda}{d}, \frac{\Lambda}{d+\alpha}<w<\frac{\Lambda}{d} \text { and } x, z<w\right\} .
$$

Now we prove that for any $\left(x_{0}, w_{0}, z_{0}\right) \in \Pi_{0}$ and $\left(x_{1}, w_{1}, z_{1}\right) \in \Pi_{0}$, there exist $T>0$ and a control function $\phi$ so that $\left(x_{\phi}(0), w_{\phi}(0), z_{\phi}(0)\right)=\left(x_{0}, w_{0}, z_{0}\right)$ and $\left(x_{\phi}(T), w_{\phi}(T), z_{\phi}(T)\right)=\left(x_{1}, w_{1}, z_{1}\right)$.

In following way we construct the function $\phi$. First, we choose a positive constant $T$ and a differentiable function $w_{\phi}:[0, T] \rightarrow\left(\frac{\Lambda}{d+\alpha}, \frac{\Lambda}{d}\right)$, so that $w_{\phi}(0)=w_{0}, w_{\phi}(T)=w_{1}, w_{\phi}^{\prime}(0)=g_{2}\left(x_{0}, w_{0}, z_{0}\right)=w_{0}^{d}, w_{\phi}^{\prime}(T)=$ $g_{2}\left(x_{1}, w_{1}, z_{1}\right)=w_{T}^{d}$ and

$$
\Lambda-(d+\alpha) w_{\phi}(t)<w_{\phi}^{\prime}<\Lambda-d w_{\phi}(t) \text { for } t \in[0, T] .
$$


We divide the construction of function $w_{\phi}$ into three intervals $[0, \varepsilon],[\varepsilon, T-\varepsilon]$ and $[T-\varepsilon, T]$, where $0<\varepsilon<\frac{T}{2}$. Let $\eta=\frac{1}{2} \min \left\{w_{0}-\frac{\Lambda}{d+\alpha}, w_{1}-\frac{\Lambda}{d+\alpha}, \frac{\Lambda}{d}-w_{0}, \frac{\Lambda}{d}-w_{1}\right\}$.

When $w_{\phi} \in\left(\frac{\Lambda}{d+\alpha}+\eta, \frac{\Lambda}{d}-\eta\right)$, we have

$$
\Lambda-(d+\alpha) w_{\phi}(t)<-(d+\alpha) \eta<0 \text { and } \Lambda-d w_{\phi}(t)>d \eta>0 \text { for } t \in[0, T] .
$$

In view of (16) we construct a $C^{2}$ function $w_{\phi}:[0, \varepsilon] \rightarrow\left(\frac{\Lambda}{d+\alpha}+\eta, \frac{\Lambda}{d}-\eta\right)$, so that

$$
w_{\phi}(0)=w_{0}, w_{\phi}^{\prime}(0)=w_{0}^{d}, w_{\phi}^{\prime}(\varepsilon)=0
$$

and for $t \in[0, \varepsilon]$ have $w_{\phi}$ satisfies (15). Similarly, we construct a $C^{2}$ function $w_{\phi}:[T-\varepsilon, T] \rightarrow$ $\left(\frac{\Lambda}{d+\alpha}+\eta, \frac{\Lambda}{d}-\eta\right)$, so that

$$
w_{\phi}(T)=w_{1}, w_{\phi}^{\prime}(T)=w_{T}^{d}, w_{\phi}^{\prime}(T-\varepsilon)=0
$$

and for $t \in[T-\varepsilon, T]$ have $w_{\phi}$ satisfies $(15)$.

Taking $T$ large enough, we extend the function $w_{\phi}:[0, \varepsilon] \cap[T-\varepsilon, T] \rightarrow\left(\frac{\Lambda}{d+\alpha}+\eta, \frac{\Lambda}{d}-\eta\right)$ to a $C^{2}$ function $w_{\phi}$ which defined on the whole interval $[0, T]$ so that

$$
\Lambda-(d+\alpha) w_{\phi}(t)<-(d+\alpha) \eta<w_{\phi}^{\prime}(t)<d \eta<\Lambda-d w_{\phi}(t) \text { for } t \in[\varepsilon, T-\varepsilon],
$$

Consequently, we get function $w_{\phi}$ that satisfies (15) on $[0, T]$. Therefore, there exists two $C^{1}$ functions $x_{\phi}$ and $z_{\phi}$ which meet the second and third equations of (13) and we can confirm a continuous function $\phi$ from the first equation of (13). This proof is completed.

Lemma 3.6. Assume that $R_{0}^{s}>1$. For every density $g$ and the semigroup $\{P(t)\}_{t \geq 0}$, we get $\lim _{t \rightarrow \infty} \iiint_{\Pi} P(t) g(x, y, z) d x d y d z=1$, where $\Pi$ is given in $(10)$.

Proof. Let $Z_{t}=S_{t}+I_{t}+R_{t}$. Then model (2) can be replaced by

$$
\left\{\begin{array}{l}
d S_{t}=g_{1}\left(S_{t}, Z_{t}, Q_{t}\right) d t-\phi \sigma S_{t}\left(Z_{t}-S_{t}-Q_{t}\right) d B_{t}, \\
d Z_{t}=g_{2}\left(S_{t}, Z_{t}, Q_{t}\right) d t \\
d Q_{t}=g_{3}\left(S_{t}, Z_{t}, Q_{t}\right) d t
\end{array}\right.
$$

where $g_{1}(x, w, z), g_{2}(x, w, z)$ and $g_{3}(x, w, z)$ are defined in $(14)$. Since $\left(S_{t}, Z_{t}, Q_{t}\right)$ is a positive solution of model (2) with probability one, we get

$$
\Lambda-(d+\alpha) Z_{t}<\frac{d Z_{t}}{d t}<\Lambda-d Z_{t}, t \in(0, \infty), \text { a.s.. }
$$

Now we prove that for almost every $\omega \in \Omega$, there exists $t_{0}=t_{0}(\omega)$ such that

$$
\frac{\Lambda}{d+\alpha}<Z_{t}(\omega)<\frac{\Lambda}{d}, \text { for } t>t_{0} .
$$

According to the position of initival value $Z_{0}$ we consider three possible cases:

(a) $Z_{0} \in\left(\frac{\Lambda}{d+\alpha}, \frac{\Lambda}{d}\right)$. On this occasion, our claim is obvious from (18).

(b) $Z_{0} \in\left(0, \frac{\Lambda}{d+\alpha}\right)$. Assume that the assertion does not hold. Then there exists $\Omega^{\prime} \subset \Omega$ increases with $\operatorname{Prob}\left(\Omega^{\prime}\right)>0$ such that $Z_{t}(\omega) \in\left(0, \frac{\Lambda}{d+\alpha}\right), \omega \in \Omega^{\prime}$. It follows from (18) that for any $\omega \in \Omega^{\prime}, Z_{t}(\omega)$ is increase in the strict sense on $[0, \infty)$. Therefore, $\lim _{t \rightarrow \infty} Z_{t}(\omega)=\frac{\Lambda}{d+\alpha}, \omega \in \Omega^{\prime}$. From the third equality of (17), we obtain $\lim _{t \rightarrow \infty} S_{t}(\omega)=\lim _{t \rightarrow \infty} Q_{t}(\omega)=0, \omega \in \Omega^{\prime}$, hence, $\lim _{t \rightarrow \infty} I_{t}(\omega)=\frac{\Lambda}{d+\alpha}, \omega \in \Omega^{\prime}$. By Itô's formula, we get

$$
d \ln I_{t}=\left(\beta S_{t}-(d+\alpha+\delta+\gamma)-\frac{1}{2} \sigma^{2} S_{t}^{2}\right) d t+\sigma S_{t} d B_{t}
$$


It yields

$$
\frac{\ln I_{t}-\ln I_{0}}{t}=\frac{1}{t} \int_{0}^{t}\left(\beta S_{s}-(d+\alpha+\delta+\gamma)-\frac{1}{2} \sigma^{2} S_{s}^{2}\right) d s+\frac{1}{t} \int_{0}^{t} \sigma S_{s} d B_{s}
$$

In view of $\frac{1}{t} \int_{0}^{t} \sigma^{2} S_{s}^{2} d s \leq \sigma^{2} \frac{\Lambda^{2}}{d^{2}}<+\infty$, by the strong law of large numbers for martingales [9], one has $\lim _{t \rightarrow \infty} \frac{1}{t} \int_{0}^{t} \sigma S_{s} d B_{s}=0$. a.s. Therefore, we have

$$
\lim _{t \rightarrow \infty} \frac{1}{t} \int_{0}^{t}\left(\beta S_{s}-(d+\alpha+\delta+\gamma)-\frac{1}{2} \sigma^{2} S_{s}^{2}\right) d s+\frac{1}{t} \int_{0}^{t} \sigma S_{s} d B_{s}=-(d+\alpha+\delta+\gamma)
$$

Since $S_{t}$ and $I_{t}$ are continuous. This contradicts the hypothesis that $\lim _{t \rightarrow \infty} \frac{\ln I_{t}-\ln I_{0}}{t}=0$ and the assertion established.

(c) $Z_{0} \in\left(\frac{\Lambda}{d},+\infty\right)$. By contradiction and similar parameters to (b), we assume that there exists $\Omega^{\prime} \subset \Omega$ with $\operatorname{Prob}\left(\Omega^{\prime}\right)>0$ so that $\lim _{t \rightarrow \infty} Z_{t}(\omega)=\frac{\Lambda}{d}, \omega \in \Omega^{\prime}$.

On account of the second and third equalities for (17), we get, for any $\omega \in \Omega^{\prime}$,

$$
\begin{aligned}
& Z_{t}(\omega)=e^{-(d+\alpha) t}\left(Z_{0}+\int_{0}^{t} e^{(d+\alpha) s}\left(\Lambda+\alpha S_{s}\right) d s\right) \\
& Q_{t}(\omega)=e^{-(d+\alpha+\varepsilon+\delta) t}\left(Q_{0}+\delta \int_{0}^{t} e^{(d+\alpha+\varepsilon+\delta) s}\left(Z_{s}-S_{s}\right) d s\right)
\end{aligned}
$$

hence, for any $\omega \in \Omega^{\prime}, \lim _{t \rightarrow \infty} S_{t}(\omega)=\frac{\Lambda}{d}, \lim _{t \rightarrow \infty} I_{t}(\omega)=\lim _{t \rightarrow \infty} Q_{t}(\omega)=0$,

$$
\begin{aligned}
\lim _{t \rightarrow \infty} \frac{1}{t} \ln I_{t}-\ln I_{0} & =\lim _{t \rightarrow \infty}\left(\frac{1}{t} \int_{0}^{t}\left(\beta S_{s}-(d+\alpha+\delta+\gamma)-\frac{1}{2} \sigma^{2} S_{s}^{2}\right) d s+\frac{1}{t} \int_{0}^{t} \sigma S_{s} d B_{s}\right) \\
& =\beta \frac{\Lambda}{d}-(d+\alpha+\delta+\gamma)-\frac{1}{2} \sigma^{2} \frac{\Lambda^{2}}{d^{2}} \\
& =(d+\alpha+\delta+\gamma)\left(R_{0}^{s}-1\right) \\
& >0, \text { a.s. on } \Omega^{\prime} .
\end{aligned}
$$

This contradicts the hypothesis that $\lim _{t \rightarrow \infty} I_{t}=0$ a.s. and the proof is completed.

Remark 3.7. From Lemmas 3.5 and 3.6, we can get that if there exists a stationary solution $u_{*}$ for the Fokker-Planck equation (4), then supp $u_{*}=\Pi$.

Lemma 3.8. Assume that $R_{0}^{s}>1$. The semigroup $\{P(t)\}_{t \geq 0}$ is sweeping or asymptotically stable as for the compact sets.

Proof. From Lemma 3.4, $\{P(t)\}_{t \geq 0}$ is an integral Markov semigroup respect to a continuous kernel $k\left(t, x, y, z, x_{0}, y_{0}, z_{0}\right)$ for $t>0$. Then, there exists a density $u(x, y, z, t)$ which satisfies (4) for distribution of $\left(S_{t}, I_{t}, Q_{t}\right)$. According to Lemma 3.6, it follows that it is sufficient to study impose restrictions on the semigroup $\{P(t)\}_{t \geq 0}$ to the space $L^{1}(\Pi)$. From Lemma 3.5, for every $f \in \mathbb{D}$, we have $\int_{0}^{\infty} P(t) f d t=$ 0 , a.s. on $\Pi$.

According to Lemma 2.1 the desired result follows.

The semigroup $\{P(t)\}_{t \geq 0}$ is asymptotically stable which is shown by the following lemmas.

Lemma 3.9. Assume that $R_{0}^{s}>1$ and $\sigma^{2}<\min \left\{A_{1}, A_{2}, A_{3}\right\}$, where $\lambda_{1}=\frac{2 d+\alpha}{\varepsilon}, \lambda_{2}=\frac{\alpha}{\delta}, \lambda_{3}=$ $\frac{2 d+\alpha+\frac{2 d+\alpha}{\varepsilon}(2 d+\alpha+\delta)}{\beta}, A_{1}, A_{2}, A_{3}$ are defined as before. Then we can get the semigroup $\{P(t)\}_{t \geq 0}$ is asymptotically stable. 
Proof. By Lemma 3.8, the semigroup $\{P(t)\}_{t \geq 0}$ satisfies the Foguel alternative. In order to rule out sweeping we should construct a non-negative $C^{2}$-function $V$ and a closed set $O \in \Sigma$ so that

$$
\sup _{(S, I, Q) \in \mathbb{X} \backslash O} \mathcal{A}^{*} V<0 .
$$

We call this function Khasminskii function [28].

When $R_{0}>1$, there exists an endemic equilibrium $E^{*}$ of model (1). Then we can get

$$
\Lambda=\beta S^{*} I^{*}+d S^{*}-\gamma I^{*}-\varepsilon Q^{*}, \beta S^{*} I^{*}=(d+\alpha+\delta+\gamma) I^{*}, \delta I^{*}=(d+\alpha+\varepsilon) Q^{*} .
$$

Let

$$
\begin{aligned}
V(S, I, Q)= & \frac{1}{2}\left(S-S^{*}+I-I^{*}+Q-Q^{*}\right)^{2}+\frac{\lambda_{1}}{2}\left(S-S^{*}+I-I^{*}\right)^{2} \\
& +\frac{\lambda_{2}}{2}\left(Q-Q^{*}\right)^{2}+\lambda_{3}\left(I-I^{*}-I^{*} \ln \frac{I}{I^{*}}\right) \\
= & V_{1}+\lambda_{1} V_{2}+\lambda_{2} V_{3}+\lambda_{3} V_{4},
\end{aligned}
$$

where $\lambda_{1}, \lambda_{2}, \lambda_{3}$ are defined as in Theorem $3.3, V$ is a nonnegative $C^{2}$-function. Then

$$
\begin{aligned}
\mathcal{A}^{*} V_{1}= & \left(S-S^{*}+I-I^{*}+Q-Q^{*}\right)(\Lambda-d(S+I+Q)-\alpha(I+Q)) \\
= & -d\left(S-S^{*}\right)^{2}-(d+\alpha)\left(I-I^{*}\right)^{2}-(d+\alpha)\left(Q-Q^{*}\right)^{2}-(2 d+\alpha)\left(S-S^{*}\right)\left(I-I^{*}\right) \\
& -(2 d+2 \alpha)\left(I-I^{*}\right)\left(Q-Q^{*}\right)-(2 d+\alpha)\left(S-S^{*}\right)\left(Q-Q^{*}\right), \\
\mathcal{A}^{*} V_{2}= & \left(S-S^{*}+I-I^{*}\right)(\Lambda-d(S+I)-\alpha I-\delta I+\varepsilon Q) \\
= & -d\left(S-S^{*}\right)^{2}-(d+\alpha+\delta)\left(I-I^{*}\right)^{2}-(2 d+\alpha+\delta)\left(S-S^{*}\right)\left(I-I^{*}\right) \\
& +\varepsilon\left(I-I^{*}\right)\left(Q-Q^{*}\right)+\varepsilon\left(S-S^{*}\right)\left(Q-Q^{*}\right), \\
\mathcal{A}^{*} V_{3}= & \frac{1}{2} \sigma^{2} S^{2} I^{*}+\beta\left(S-S^{*}\right)\left(I-I^{*}\right), \\
\mathcal{A}^{*} V_{4}= & \left(Q-Q^{*}\right)(\delta I-(d+\alpha+\varepsilon) Q) \\
= & -(d+\alpha+\varepsilon)\left(Q-Q^{*}\right)^{2}+\delta\left(I-I^{*}\right)\left(Q-Q^{*}\right),
\end{aligned}
$$

Hence, we have

$$
\begin{aligned}
\mathcal{A}^{*} V= & \mathcal{A}^{*} V_{1}+\lambda_{1} \mathcal{A}^{*} V_{2}+\lambda_{2} \mathcal{A}^{*} V_{3}+\lambda_{3} \mathcal{A}^{*} V_{4} \\
= & -d\left(1+\lambda_{1}\right)\left(S-S^{*}\right)^{2}-\left(d+\alpha+\lambda_{1}(d+\alpha+\delta)\right)\left(I-I^{*}\right)^{2} \\
& -\left(d+\alpha+\lambda_{2}(d+\alpha+\varepsilon)\right)\left(Q-Q^{*}\right)^{2}+\frac{1}{2} \sigma^{2} I^{*} \lambda_{3} S^{2} \\
= & -\frac{1}{2 \varepsilon \beta}\left(2 \varepsilon \beta d+2 \beta(2 d+\alpha) d-\sigma^{2} I^{*}(2 d+\alpha)(\varepsilon+2 d+\alpha+\delta)\right) \\
& \left(S-\frac{2 \varepsilon \beta d \frac{2 d+\alpha+\varepsilon}{\varepsilon}}{2 \varepsilon \beta d+2 \beta(2 d+\alpha) d-\sigma^{2} I^{*}(2 d+\alpha)(\varepsilon+2 d+\alpha+\delta)} S^{*}\right)^{2} \\
& -\left(d+\alpha+\frac{2 d+\alpha}{\varepsilon}(d+\alpha+\delta)\right)\left(I-I^{*}\right)^{2}-\left(d+\alpha+\frac{\alpha}{\varepsilon}(d+\alpha+\delta)\right)\left(Q-Q^{*}\right)^{2} \\
& +\frac{\left(d+d \frac{2 d+\alpha}{\varepsilon}\left(2 \varepsilon \beta(d-1)+\sigma^{2} I^{*}(2 d+\alpha)(\varepsilon+2 d+\alpha+\delta)\right)\right)}{2 \varepsilon \beta+2 \beta(2 d+\alpha) d-\sigma^{2} I^{*}(2 d+\alpha)(\varepsilon+2 d+\alpha+\delta)} S^{*^{2}} \\
= & -a_{1}\left(S-b_{1} S^{*}\right)^{2}-a_{2}\left(I-I^{*}\right)^{2}-a_{3}\left(Q-Q^{*}\right)^{2}+a_{4} \cdot
\end{aligned}
$$


In view of the conditions, we can get

$$
\begin{aligned}
& \frac{\left(d+d \frac{2 d+\alpha}{\varepsilon}\left(2 \varepsilon \beta(d-1)+\sigma^{2} I^{*}(2 d+\alpha)(\varepsilon+2 d+\alpha+\delta)\right)\right)}{2 \varepsilon \beta+2 \beta(2 d+\alpha) d-\sigma^{2} I^{*}(2 d+\alpha)(\varepsilon+2 d+\alpha+\delta)} S^{*^{2}} \\
& <\min \left\{\frac{2 \varepsilon \beta d^{2} \frac{(2 d+\alpha+\varepsilon)^{2}}{\varepsilon^{2}}}{2 \varepsilon \beta+2 \beta(2 d+\alpha) d-\sigma^{2} I^{*}(2 d+\alpha)(\varepsilon+2 d+\alpha+\delta)} S^{*^{2}}, d+\alpha+\lambda_{1}(d+\alpha+\delta),\right. \\
& \left.d+\alpha+\lambda_{2}(d+\alpha+\varepsilon)\right\} .
\end{aligned}
$$

It then follows that the ellipsoid $-a_{1}\left(S-b_{1} S^{*}\right)^{2}-a_{2}\left(I-I^{*}\right)^{2}-a_{3}\left(Q-Q^{*}\right)^{2}+a_{4}=0$. Lies entirely in $\mathbb{X}$. Therefore there exists a closed set $O \in \Sigma$ which contains this ellipsoid and $c>0$ such that

sup $\mathcal{A}^{*} V \leq-c<0$.Using similar arguments to those in [28], the presence of a Khasminskii $(S, I, Q) \in \mathbb{X} \backslash O$

function means that the semigroup is not sweeping from the set $O$. The proof is completed.

Remark 3.10. It should be noted that the condition of Lemma 3.9 suggests ,if we wanna have a stationary distribution, that the amplitude of the noise should not be too large .

Therefore, together with Lemmas 3.8 and 3.9, we obtain Theorem 3.3.

\section{Numerical Simulations}

In this section, we present a numerical example to illustrate the analytical results. In the following, we assume that $\Lambda=0.4, \delta=0.005, \epsilon=0.5, \alpha=0.01, \beta=0.2, d=0.2, \gamma=0.04$. It is easy to calculate $R_{0}=\frac{\Lambda \beta}{d(\gamma+\delta+d+\alpha)} \approx 1.626>1$. From [5] we obtain that the model (1) has one endemic equilibrium. By the above parameters, we can obtain the locally stable endemic equilibrium $E^{*}=\left(S^{*}, I^{*}, Q^{*}\right)$, and unstable $E_{0}$. But for the stochastic model $(2)$, there exists a stationary distribution. When $\mathrm{S}(0)=0.3, \mathrm{I}(0)$ $=0.3, \mathrm{Q}(0)=0.4$ and noisy intensity $\sigma=0.1$. By calculating, it satisfies the condition of Theorem 3.3. In other words, there exists a stationary distribution shown in Figure 2. Using the Kolmogorov-Smirnov test to compare the distributions of the stochastic solution paths of $S, I$ and $Q$ at $t=4000,4500,5000$ based on 5000 sample paths with $S(0)=0.3, I(0)=0.3, Q(0)=0.4$ with noisy intensity $\sigma=0.1 .5000$ sample paths of stochastic model were run until $t_{\text {end }}=5000$.

In Figure 1, we plot the boxplot of the 5000 sample paths of $S, I$ and $Q$ at $t=4000,4500,5000$, respectively, and find that the mean, minimum and maximum values at $t=4000,4500,5000$ are all on a straight line of $S, I$ and $Q$, respectively. Further, putting together the probability density functions at $t=4000,4500,5000$ of $S, I$ and $Q$, respectively, we find that the curves almost overlap (see Figure 2 and Figure 3). Therefore, the stochastic model has a unique asymptotic stability stationary distribution. Use 10-point type for the name(s) of the author(s) and 9-point type for the address(es) and the abstract. For the main text, please use 10-point type and single-line spacing. We recommend the use of Computer Modern Roman or Times. Italic type may be used to emphasize words in running text. Bold type and underlining should be avoided.
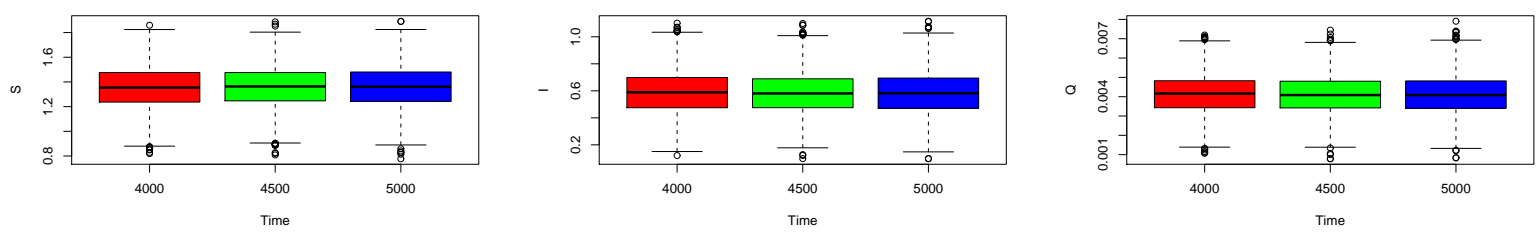

Figure 1. Boxplots of the sample paths for susceptible individuals S, infected individuals I and quarantined individuals $\mathrm{Q}$ when $\mathrm{S}(0)=0.3, \mathrm{I}(0)=0.3, \mathrm{Q}(0)=0.4$ and noisy intensity $\sigma=0.1$ based on 5000 sample paths at $\mathrm{t}=4000,4500,5000$. 

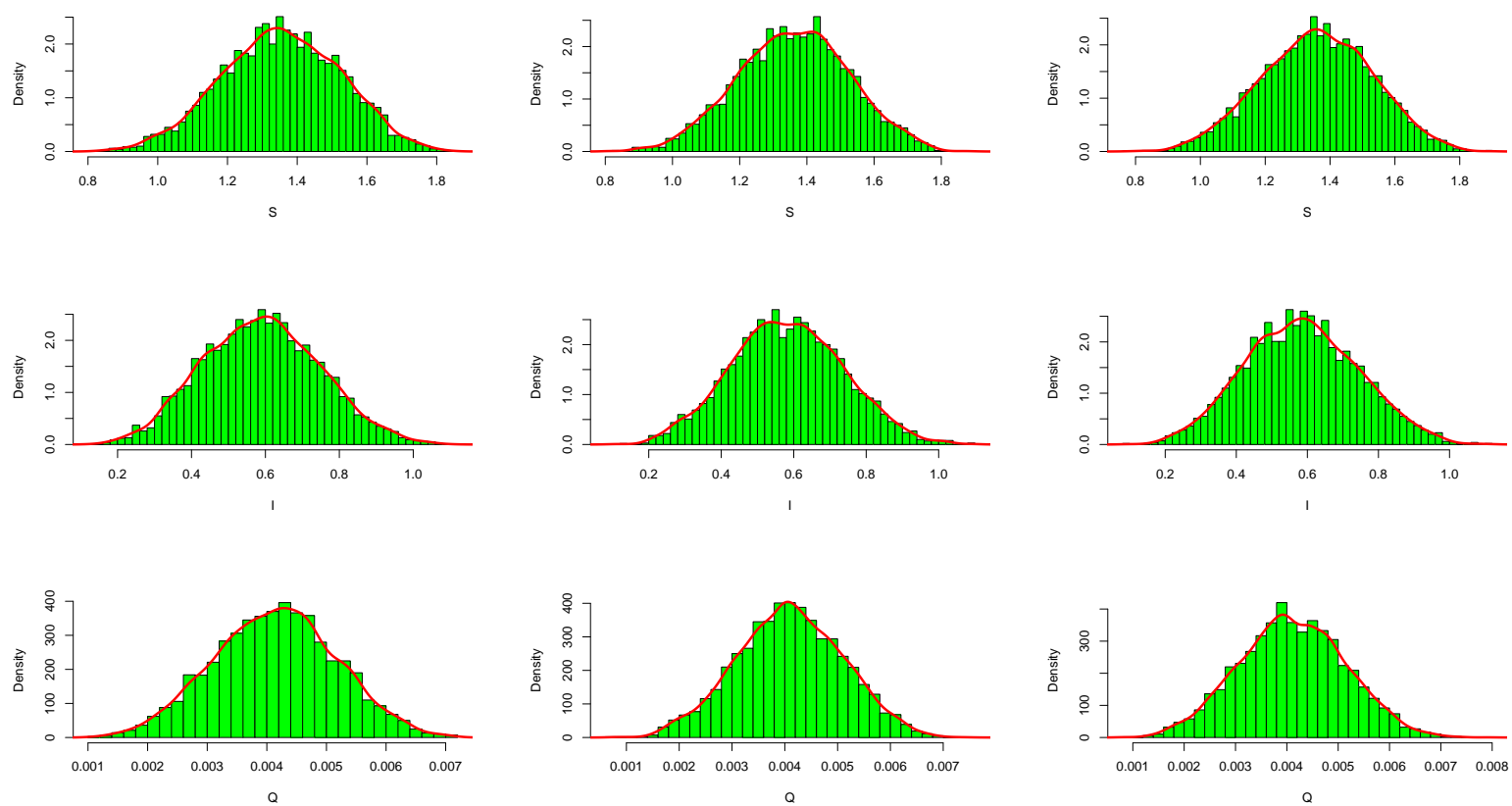

Q

Figure 2. Histogram of the probability density function for susceptible individuals $\mathrm{S}$ (first line), infected individuals $\mathrm{I}$ (second line) and quarantined individuals Q (third line) when $\mathrm{S}(0)=0.3, \mathrm{I}(0)=0.3, \mathrm{Q}(0)=0.4$ and noisy intensity $\sigma=0.1$ based on 5000 sample paths at $\mathrm{t}=4000$ (left column), $\mathrm{t}=4500$ (middle column), $\mathrm{t}=5000$ (right column).
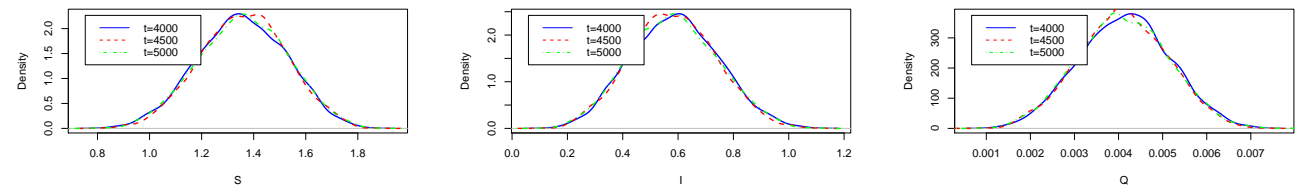

Figure 3. The probability density function for susceptible individuals $\mathrm{S}$, infected individuals I and quarantined individuals $\mathrm{Q}$ when $\mathrm{S}(0)=0.3, \mathrm{I}(0)=0.3, \mathrm{Q}(0)=0.4$ and noisy intensity $\sigma=0.1$ based on 5000 sample paths at $\mathrm{t}=4000,4500,5000$.

\section{References}

1. W.H. McNeill, Plagues and Peoples, Anchor, Garden City, NY, 1976.

2. Z.E. Ma, Y.C. Zhou, W.D. Wang, Z. Jin, Mathematical modeling and research of infectious disease dynamics, Beijing: Science Press, (2006).

3. H.W. Hethcote, Qualitative analyses of communicable disease models, Math. Biosci, 28 (1976) 335.

4. L. Wu, Z. Feng, Homoclinic bifurcation in an SIQR model for childhood diseases, J. Differ. Equations, 168 (2000) 150.

5. H. Herbert, Z. Ma, S. Liao, Effects of quarantine in six endemic models for infectious diseases, Math. Biosci, 180 (2002) 141-160.

6. S. Spencer, Stochastic epidemic models for emerging diseases, PhD thesis, University of Nottingham, 2008.

7. J.R. Beddington, R.M. May, Harvesting natural populations in a randomly fluctuating environment, Science, 197 (4302) (1977) 463-465.

8. J.E. Truscott, C.A. Gilligan, Response of a deterministic epidemiological system to a stochastically varying environment, Proc. Natl. Acad. Sci. USA, 100 (15) (2003) 9067-9072.

9. X. Mao, Stochastic Differential Equations and Their Applications, Horwood, Chichester, 1997.

10. R. Durrett, Stochastic spatial models, SIAM Rev, 41 (4) (1999) 677-718. 
11. B. Dennis, Allee effects in stochastic populations, Oikos, 96 (3) (2002) 389-401.

12. X. Mao, S. Sabanis, E. Renshaw, Asymptotic behaviour of the stochastic Lotka-Volterra model, J. Math. Anal. Appl, 287 (1) (2003) 141-156.

13. X. Mao, G. Marion, E. Renshaw, Environmental Brownian noise suppresses explosions in population dynamics, Stochastic Process. Appl, 97 (1) (2002) 95-110.

14. P.S. Mandal, M. Banerjee, Stochastic persistence and stationary distribution in a Holling-Tanner type prey-predator model, Phys. A, 391 (4) (2012) 1216-1233.

15. L.J.S. Allen, P. Driessche, Stochastic epidemic models with a backward bifurcation, Math. Biosci. Eng, 3 (3) (2006) 445-458.

16. L.J.S. Allen, An introduction to stochastic epidemic models, in: Math. Epidem, Springer, (2008) 81-130.

17. T. Komorowski, J. Tyrcha, Asymptotic properties of some Markov operators, Bull. Pol. Acad. Sci. Math, 37 (1-6) (1989) 221-228.

18. R. Rudnicki, On asymptotic stability and sweeping for Markov operators, Bull. Pol. Acad. Sci. Math, 43 (3) (1995) 245-262.

19. R. Rudnicki, Long-time behaviour of a stochastic prey-predator model, Stochastic Process. Appl, 108 (1) (2003) 93-107.

20. A. Lasota, M.C. Mackey, Chaos, Fractals, and Noise: Stochastic Aspects of Dynamics, Springer, 97 (1994).

21. L.P. Kadanoff, Statistical Physics: Statics, Dynamics, and Renormalization, World Scientific, (2000).

22. R. Rudnicki, K. Pichor, M. Tyran-Kaminska, Markov Semigroups and Their Applications, Lecture Notes in Phys, 597 (2002) 215-238.

23. C. Chen, Y. Kang, The asymptotic behavior of a stochastic vaccination model with backward bifurcation, Applied Mathematical Modelling, (2016) 1-18.

24. H.R. Thieme, Convergence results and a Poincaré-Bendixson trichotomy for asymptotically autonomous differential equations, Journal of Mathematical Biology, 30 (7) (1992) 755-763.

25. D.R. Bell, The Malliavin Calculus, Dover Publications, (2006).

26. D.W. Stroock, S.R.S. Varadhan, On the support of diffusion processes with applications to the strong maximum principle, in: Proceedings of the Sixth Berkeley Symposium on Mathematical Statistics and Probability, Univ. California, Berkeley, Calif, 1970/1971 3 (1972) 333-359.

27. G.B. Arous, R. Léandre, Décroissance exponentielle du noyau de la chaleur sur la diagonale (II), Probab. Theory Related Fields, 90 (3) (1991) 377-402.

28. K. Pichór, R. Rudnicki, Stability of Markov semigroups and applications to parabolic systems, J. Math. Anal. Appl, 215 (1) (1997) 56-74. 